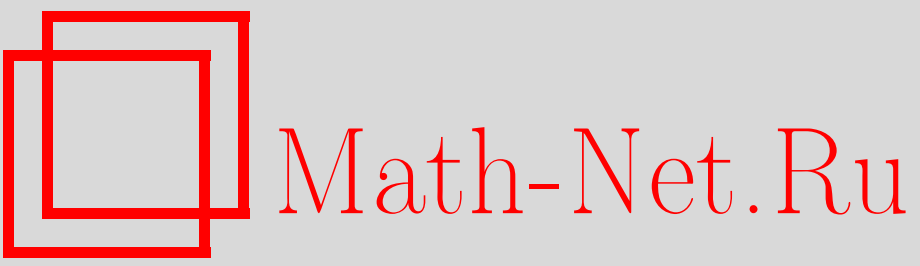

Л. Д. Пустыльников, Бесконечномерные обобщенные цепные дроби, квадратичные вычеты и невычеты и эргодическая теория, УМH, 1997, том 52, выпуск 2, 183-184

DOI: https://doi.org/10.4213/rm842

Использование Общероссийского математического портала Math-Net.Ru подразумевает, что вы прочитали и согласны с пользовательским соглашением

http: //www . mathnet.ru/rus/agreement

Параметры загрузки:

IP: 54.172 .240 .79

26 апреля 2023 г., 14:33:47 


\title{
БЕСКОНЕЧНОМЕРНЫЕ ОБОБЩЕННЫЕ ЦЕПНЫЕ ДРОБИ, КВАДРАТИЧНЫЕ ВЫЧЕТЫ И НЕВЫЧЕТЫ И ЭРГОДИЧЕСКАЯ ТЕОРИЯ
}

\author{
Л. Д. Пустыльников
}

В работах [1], [2] было введено понятие $(A, \omega)$-цепной дроби произволшного вещественного $n$-мерного вектора, обобщающее обьчную цепную дробь действительного числа и зависящее от некоторого преобразования $A \quad n$-мерного пространства и вектора $\omega$, принадлежащего единичному $n$-мерному тору. В настоящей работе мы используем конструкцию $(A, \omega)$-цепной дроби из [1] и [2] для определения $(A, p, \omega)$-цепной дроби произвольного бесконечномерного вектора $x=\left(x_{1}, x_{2}, \ldots\right)$ с целочисленными координатами $x_{n} \quad(n=1,2, \ldots)$, устанавливаем основные свойства этих дробей (теоремы 1,2 ) и применяем их к классической проблеме [4] о распределении квадратичных вычетов и невычетов по простому модулю (теорема 3). Доказательство использует эргодическую теорию (теорема 4).

Введем следующие объекты:

1) последовательность $p=\left(p_{1}, p_{2}, \ldots\right)$, состоящую из бесконечного числа попарно различных, взаимно простых натуральных чисел $p_{n}(n=1,2, \ldots)$;

2 ) последовательность $\gamma=\left(\gamma_{1}, \gamma_{2}, \ldots\right)$, состоящую из целых чисел $\gamma_{n}$, у которой при любом $n=1,2, \ldots$ число $\gamma_{n}$ взаимно просто с $p_{n}$;

3 ) дискретную окружность $S_{n}$, состоящую из вычетов $0,1, \ldots, p_{n}-1$ по модулю $p_{n}$, и такую меру $\mu_{n}$ на $S_{n}$, что мера каждого элемента из $S_{n}$ равна $p_{n}^{-1}$;

4) тор $T$, являющийся декартовым произведением окружностей $S_{n}$ по всем $n=1,2, \ldots$, точка которого есть такой вектор $\omega=\left(\omega_{1}, \omega_{2}, \ldots\right)$, что $\omega_{n} \in S_{n} \quad(n=1,2, \ldots)$, и меру теs на $T$, являющуюся произведением мер $\mu_{n}$ по всем $n$;

5 ) преобразование $A$, переводящее вектор $x=\left(x_{1}, x_{2}, \ldots\right)$ в вектор $x^{\prime}=A x=\left(x_{1}^{\prime}, x_{2}^{\prime}, \ldots\right)$ с координатами

$$
x_{n}^{\prime}=x_{n}+\gamma_{n} \quad(n=1,2, \ldots)
$$

6) преобразование $\widehat{A}$ тора $T$, переводящее вектор $\omega=\left(\omega_{1}, \omega_{2}, \ldots\right) \in T$ в вектор $\omega^{\prime}=\widehat{A} \omega=$ $\left(\omega_{1}^{\prime}, \omega_{2}^{\prime}, \ldots\right)$ с координатами

$$
\omega_{n}^{\prime}=\omega_{n}-\gamma_{n} \quad \bmod p_{n} \quad(n=1,2, \ldots) ;
$$

7) для любого вектора $x=\left(x_{1}, x_{2}, \ldots\right)$ вводим векторы

$$
[x]=\left([x]_{1},[x]_{2}, \ldots\right), \quad\{x\}=\left(\{x\}_{1},\{x\}_{2}, \ldots\right),
$$

у которых при $n=1,2, \ldots$ координата $[x]_{n}$ есть целая часть числа $x_{n} / p_{n}$, a $\{x\}_{n}=x_{n}-p_{n}[x]_{n}$.

Для любого вектора $\omega \in T$ введем $(A, p, \omega)$-цепную дробь произвольного бесконечномерного вектора $x$ с целыми координатами, которая может быть как конечной, так и бесконечной, обозначается через $x=\left[q^{(0)}, \ldots, q^{(\nu)}\right]_{A, p, \omega}$, если она конечна, через $x=\left[q^{(0)}, q^{(1)}, \ldots\right]_{A, p, \omega}$, если она бесконечна, и определяется с помощью рекуррентной процедуры, состоящей из конечного или бесконечного числа шагов, а на произвольном шаге с номером $k(k=0,1,2, \ldots)$ осуществляются следующие три операции:

а) определяется вектор $q^{(k)}=\left[x^{(k)}\right]$, где $x^{(k)}$ - вектор, полученный на $(k-1)$-м шаге, а $x^{(0)}=x$

б) сравниваются векторы $\delta^{(k)}=\left\{x^{(k)}\right\}$ и $\omega$;

в) процесс прекращается, если $\delta^{(k)}=\omega$, и определяется вектор $x^{(k+1)}=A \delta^{(k)}$, если $\delta^{(k)} \neq \omega$

Для $(A, p, \omega)$-цепных дробей справедливы теоремы $1,2,3$ из [1]. Кроме того, справедливы следующие теоремы.

Tеорема 1. Пусть $\Omega \subset T$, mes $\Omega>0$. Тогда для почти всех $x \in T$ (в смицсле мерь теs нат) существует такое $\omega=\omega(x) \in \Omega$, что $(A, p, \omega)$-цепная дробь вектора х конечна. 
ОПРЕДЕЛЕниЕ 1 . Пусть $x=\left[q^{(0)}, q^{(1)}, \ldots\right]_{A, p, \omega}$. При $\nu=0,1, \ldots$ введем $(A, p, \omega)$-подходящие дроби $S^{(\nu)}=\left[q^{(0)}, \ldots, q^{(\nu)}\right]_{A, p, \omega}: S^{(\nu)}=\left(S_{1}^{(\nu)}, S_{2}^{(\nu)}, \ldots\right)$ - бесконечномерный вектор, у которого при $n=0,1, \ldots$ координата

$$
S_{n}^{(\nu)}=q_{n}^{(0)} p_{n}+\left(\ldots\left(-\gamma_{n}+q_{n}^{(\nu-1)} p_{n}+\left(-\gamma_{n}+q_{n}^{(\nu)} p_{n}+\omega_{n}\right)\right) \ldots\right)
$$

где $q_{n}^{(k)}$ и $\omega_{n}(k=0, \ldots, \nu)$ - координаты векторов

$$
q^{(k)}=\left(q_{1}^{(k)}, q_{2}^{(k)}, \ldots\right), \quad \omega=\left(\omega_{1}, \omega_{2}, \ldots\right) .
$$

Теорема 2. Пусть дана бесконечная $(A, p, \omega)$-цепная дробь вектора $x=\left(x_{1}, x_{2}, \ldots\right)$, $S^{(\nu)}=\left(S_{1}^{(\nu)}, S_{2}^{(\nu)}, \ldots\right)-(A, p, \omega)$-подходящие дроби вектора $x$. Тогда $x$ есть предельная точка последовательности $S^{(\nu)}$ в следующем смысле: для каждого натурального $N$ существует такое $\nu$, что у вектора $S^{(\nu)}$ координата $S_{n}^{(\nu)}=x_{n}$, если $1 \leqslant n \leqslant N$.

ОпредЕлЕниЕ 2 . Введем преобразование $A^{*}$, которое совпадает с преобразованием $A$, если в его определении положить $\gamma_{n}=1(n=1,2, \ldots)$.

Теорема 3. Пусть $\psi(n)$ - произвольная вещественная функция такая, что при всех $n \geqslant 1 \psi(n) \neq 0, \lim _{n \rightarrow \infty} \psi(n)=\infty u\left|\psi^{k}(n)\right| \leqslant c \sqrt{n}$, әде $k-$ натуральное число, $k$ ис - константы, не зависящие от $n ; p=\left(p_{1}, p_{2}, \ldots\right)$ - последовательность, состоящая из попарно различных простых чисел $p_{n}(n=1,2, \ldots)$ такая, что $\sum_{n=1}^{\infty}\left|\psi\left(p_{n}\right)\right|^{-k}<\infty$; $x=\left(x_{1}, x_{2}, \ldots\right) \in T$. Тогда существ ует и конструктивно строится множество $\Omega \subset T$, такое что выполняются следующие утверждения:

1) если существует такой вектор $\omega \in \Omega$, что $\left(A^{*}, p, \omega\right)$-цепная дробь вектора х конечна и имеет вид $x=\left[q^{(0)}, \ldots, q^{(\nu)}\right]_{A^{*}, p, \omega}$, то существует такая константа $d$, зависящая только от р и $\psi(n)$, что при всех $n=1,2, \ldots$ среди челых чисел $z$, расположенных в области

$$
x_{n}<z<x_{n}+d+\nu+\psi\left(p_{n}\right),
$$

содержатся квадратичные вычет и невычет по модулю $p_{n}$, и множество векторов $x \in T$, для которых выполнено это утверждение, имеет в $T$ дополнение, мера теs которого равна нулю;

2) если при $\omega \in \Omega$ вектор $x$ разлагается в бесконечную $\left(A^{*}, p, \omega\right)$-цепную дробь, $a S^{(\nu)}=\left(S_{1}^{(\nu)}, S_{2}^{(\nu)}, \ldots\right)-$ такая $\left(A^{*}, p, \omega\right)$-подходящая дробь, что $n p u 1 \leqslant n \leqslant N$ выполняются равенства $S_{n}^{(\nu)}=x_{n}$, то в области (1) содер жатся квадратичные вычет и невьчет по модулю $p_{n}$, если $1 \leqslant n \leqslant N$.

ТеОрема 4. Преобразование $\widehat{A}$ на торе $T$ строго әргодично относительно меры теs.

Доказательство теоремы 3 основано на доказательстве теоремш 2 из [3], а доказательство теоремы 4 использует доказательство теоремы 1 из [3] и [5].

\section{СПИСОК ЛИТЕРАТУРЫ}

[1] Пустыльников Л. Д. // Матем. заметки. 1994. Т. 56. №6. С. 144-147. [2] Pustylnikov L. D. Ergodic Theory and some Number Theory Problems. Preprint Eorshungsinstitut für Mathematik ETH. Zürich, July, 1994. [3] Пустыльников Л.Д. // УМН. 1993. Т. 48. №1. С. 185-186. [4] Гельфонд А.О., Линник Ю. В. Элементарные методы в аналитической теории чисел. М.: Изд-во физ.-мат. лит., 1962. [5] Корнфельд И. П., Синай Я. Г., Фомин С. В. Эргодическая теория. М., 1975. 